Original Article

\title{
CORRELATION OF ANTIOXIDANT LEVELS IN SYNOVIAL FLUID TO INTENSITY OF PAIN IN OSTEOARTHRITIS OF KNEE
}

\author{
Hiba Esmayil ${ }^{1} \&$ Siddharth M. Shetty ${ }^{2}$ \\ ${ }^{1}$ Intern, ${ }^{2}$ Associate Professor, Department of Orthopaedics, K. S. Hegde M edical Academy, \\ Nitte University, Mangalore - 575018, Karnataka, India. \\ Correspondence : \\ Hiba Esmayil \\ Rashas, Chirakkara P.0, Tellicherry, Kannur, Kerala - 670104, India. \\ M obile : +91 74116 42021, +91 490 2323802. E-mail : hibaesmayil@ gmail.com
}

\begin{abstract}
:
Introduction : Osteoarthritis $(\mathrm{OA})$ refers to progressive degeneration of articular cartilage with gradual painful disorganisation of the joint. Lipid peroxidation mediated by free radicals is the major mechanism of cell membrane destruction and cell damage. It is a known that there is an increased oxidative stress in $\mathrm{OA}$ and very little is known about the relation between the pain and decreased antioxidant levels. This study aimed at understanding the relation between the level of superoxide dismutase (SOD) and total antioxidant capacity (TAC) of synovial fluid in OA knee with the intensity of pain.
\end{abstract}

Materials and Methods : 30 patients with clinicoradiologically evidence of knee OA were included in the study and pain was scored using Visual Analog Scale. $1 \mathrm{ml}$ synovial fluid was aspirated from the knee and superoxide dismutase (SOD) and total antioxidant capacity (TAC) was estimated. Statistical analysis between the pain score and the SOD and the TAC was done independently by Spearman's rank correlation method and correlation coefficient was obtained.

Results : It was found that SOD and the TAC values were reduced in our subjects with mean value of $0.06477 \pm 0.044814$ and $122.51000 \pm$ 50.764829 respectively. The mean value of pain was $6.70 \pm 1.317$. A significant correlation between SOD level and pain score $(p \varangle 0.05)$ was obtained. However, the correlation between TAClevel and pain score was not significant $(p>0.05)$.

Conclusion : The study concluded that there is a positive correlation between the intensity of pain and SOD levels in the synovial fluid in OA of knee which is due to the increased free radicals produced.

Keywords: oxidative stress of the knee, Superoxide dismutase, Total antioxidant capacity

\section{Introduction:}

Osteoarthritis $(O A)$ refers to the progressive deterioration and degeneration in the structure of articular cartilage leading to formation of new bone (osteophyte) at the margins of the bone of the joint. It is a progressively disabling disease of the joints that affects up to $50 \%$ of the population with neither a clear cause nor an effective treatment ${ }^{[1]}$. Symptoms include joint pain, tenderness, stiffness, locking, and effusion. A variety of causes are

\begin{tabular}{|c|}
\hline Access this article online \\
\hline Quick Response Code \\
\hline
\end{tabular}
attributed as risk factors such as hereditary or familial, developmental, metabolic, and mechanical which may initiate the process leading to loss of cartilage and degeneration. When bone surfaces are devoid of protection by cartilage, the subchondral bone is exposed and damaged. The naked nerve endings in the bone are irritated leading to pain, spasm, decreased range of movement of the joint. The muscles atrophy and ligaments show destruction leading to laxity or contractures. There are no diagnostic laboratory tests to predict or confirm the disease in the early stages and radiographic evidence of osteoarthritis often occurs late in the disease course, only after a significant loss of cartilage tissue.

Pain is the most important symptom of osteoarthritis and is the reason why individuals seek medical treatment. Pain not only contributes to functional limitations and reduced quality of life but is also the leading cause of impaired mobility in the elderly population. This fact led us to select this group as subjects. There may also be either loss of 
stability and sometimes stiffness. Pain is generally described as a sharp ache, or a burning sensation in the muscle group. Osteoarthritis can cause a crackling noise ("crepitus") when the affected joint is moved or touched, and patients may experience muscle spasm.

Pain in the subjects is measured using the visual analog scale. This study may contribute in analyzing the role of oxidative stress in the joint synovial fluid in relation to pain, so as to understand the role of supplementation of antioxidants in the management of osteoarthritis.

The body is constantly utilizing oxygen for a myriad of vital functions. A by-product of oxygen uptake by the body is the creation of chemicals known as free radicals. Lipid peroxidation mediated by free radicals called reactive oxygen species (ROS), is the major mechanism of cell membrane destruction and cell damage in OA. As important as the rate of production of ROS is the scavenging capacity of the system relative to the production of ROS by means of antioxidants. Antioxidants include superoxide dismutase (SOD), catalase, glutathione peroxidase, Vitamin A, beta-carotene, Vitamins $\mathrm{C}$ and $\mathrm{E}$, selenium and melatonin. Some antioxidant supplements or drugs with antioxidant properties have been developed to reinforce the cellular antioxidant status. However, until now, there is no consistent evidence that additional antioxidant supply is efficient to relieve OA symptoms or to prevent structural changes in OA cartilage ${ }^{[2]}$.

The superoxide anion $\mathrm{O}^{2-}$ is the main ROS. Increased ROS production leads to tissue damage associated with inflammation. Superoxide dismutases (SODs) convert superoxide to hydrogen peroxide, which is then removed by glutathione peroxidase or catalase. Three distinct SODs are found in the human body: SOD1, or CuZnSOD, which localizes primarily to the cytosol, SOD2, or M nSOD, which is found in the mitochondria, and SOD3, or extracellular SOD (EC-SOD). EC-SOD is a secreted tetrameric glycoprotein with a positively charged heparin-binding site ${ }^{[3]}$. The active center contains copper and zinc and is homologous with SOD1, but the EC-SOD molecule is immunologically distinct, and the $\mathrm{C}$-terminal region contains a positively charged heparin-binding region. It localizes to the ECM of tissues by binding to the negatively charged proteoglycans and collagen ${ }^{[4,5]}$. In this location, it can protect the vulnerable proteins and macromolecules of the ECM from oxidant injury. This important antioxidant has been studied in the vascular, pulmonary, and neurologic systems, but not in cartilage or joints ${ }^{[3,6,7]}$.

We postulated that EC-SOD may be one of the major antioxidants in the cartilage, and its deficiency would lead to oxidant damage in the matrix and clinical disease. We studied human knee joint fluid samples to test whether there is an association between the increased pain in $\mathrm{OA}$ and the decreased EC-SOD in the joint fluid. Synovial fluid was chosen to be the sample as it is directly produced by the joints, as it would be the most accurate indicator to estimate the oxidative stress seen in $\mathrm{OA}$.

Previous studies by Krishna Mohan Surapaneni et al ${ }^{[8]}$ showed a higher oxygen-free radical production, and a decrease in antioxidant level in osteoarthritis. Sutipornpalangkul $W$ et al' $s{ }^{[9]}$ work showed that oxidative stress may have a role in its pathogenesis of knee osteoarthritis and Vitamin E supplementation may have a role in the management. M CAlindon TE et al ${ }^{[10]}$ found that high intake of antioxidant micronutrients, especially vitamin $\mathrm{C}$, may reduce the risk of cartilage loss and disease progression in $\mathrm{OA}$.

However, little is known about the possible interaction between decreased antioxidant levels and the pain reported in OA. Thus, this study shall attempt to estimate the level of SOD and total antioxidant capacity in synovial fluid of osteoarthritis patients and compare it with the intensity of pain, thus trying to establish a relationship between the two. The study will also provide scope for further research in this topic and help in identifying the role of the supplementation of antioxidants in the regression of pain.

\section{Materials and Methods:}

I 30 patients who visited the orthopedics outpatient department of the medical college with 
clinicoradiological evidence of knee osteoarthritis over a period of two months were included in the study.

I Ethical clearance was obtained from the institutional ethical committee for the conduct of study.

I Informed written consent was obtained from the patient prior to recruitment into the study.

I Visual analog scale was used for scoring the pain, this is a chart used to subjectively measure the intensity of knee pain. The visual analog scale of pain is a $100 \mathrm{~mm}$-long horizontal line, which contains word descriptions at each end (e.g.: no pain and worst pain on either sides of the line). The patients represent their perception of the intensity of pain they feel by marking a horizontal line. The visual analog scale score is measured in millimeters from the left hand end of the line to the point indicated by the patient.

I $1 \mathrm{ml}$ synovial fluid was aspirated from the knee under aseptic precautions was sent to the central research laboratory.

I Superoxide dismutase was estimated from the synovial fluid of the patients using the nitro blue tetrazolium chloride (NBT) reduction method ${ }^{[13,14]}$. The estimation of superoxide dismutase enzyme is carried out by Beauchamp and Fridovich method. The substrate used for the assay consists of nitro blue tetrazolium chloride (NBT) which reacts with superoxide anions produced upon illumination of riboflavin in the presence of methionine as an electron donor, to produce formazan which is a blue colored complex. The SOD present in the sample will act on the superoxide anions produced by riboflavin and thereby reduce the net superoxide anions in the substrate leading to decreased production of formazan manifested by decreased intensity of the blue color formed. The decrease in the formation of formazan is directly proportional to the amount of SOD in the sample, $50 \%$ decrease in the formation of formazan is taken as one unit of SOD. The standard was prepared by adding $2.5 \mathrm{~mL}$ methionine, $0.3 \mathrm{~mL}$ Riboflavin, $0.1 \mathrm{mLNBT}$ and $0.1 \mathrm{~mL}$ phosphate buffer 0.05 $\mathrm{M}$ (pH 7.8).

I Total antioxidant capacity was estimated from the synovial fluid of the patients using the phosphomolybdenum method ${ }^{[15]}$. The quantitative assay is based on the conversion of M olybdenum (M O VI) by reducing agents like antioxidants to molybdenum(M० V), which further reacts with the phosphate under acidic $\mathrm{pH}$ resulting in the formation of a green colored complex, the intensity of which can be read spectrophotometrically at $695 \mathrm{~nm}$. The standard was prepared by taking $10 \mathrm{mg}$ of Vitamin C in a $10 \mathrm{~mL}$ standard flask and making it up to the mark with distilled water. $100 \mu \mathrm{L}$ of $5 \% \mathrm{TCA}$ is added to $100 \mu \mathrm{L}$ of the sample to precipitate out the proteins in the sample; the mixture is then allowed to stand for about five minutes and centrifuged. $1 \mathrm{~mL}$ of TACreagent is added to $100 \mu \mathrm{L}$ of the clear supernatant and the mixture is then incubated in water bath at $90^{\circ} \mathrm{C}$ for 90 minutes. A blank is also maintained simultaneously by substituting $100 \mu \mathrm{L}$ of water instead of sample in the reaction mixture. Following the incubation the reaction mixture is cooled and the optical density of the greenish to bluish color formed is read at $695 \mathrm{~nm}$ against blank. The total antioxidant capacity of the sample is calculated by plotting the optical density of the test against the standard graph. The total antioxidant capacity is expressed as $\mu \mathrm{g} / \mathrm{ml}$. The data obtained was analyzed for statistical significance by spearman's rank correlation method to obtain the correlation coefficient between SOD level and the pain score, and the total antioxidant capacity and the pain score separately. A p value $<0.05$ was considered significant. Scatter diagram with line of regression was plotted.

\section{A) INCLUSION CRITERIA:}

Patients with clinicoradiological confirmation of knee osteoarthritis will be included in the study.

\section{B) EXCLUSION CRITERIA:}

- Patients with surgery around the same jo int in the past.

- Patients with inflammatory joint diseases.

- Patients on steroids or long term medications.

- Patients with pain following trauma. 
C) EXPERIMENTALDESIGN:

30 Patients with knee osteoarthritis with radiological confirmation

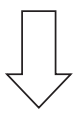

The intensity of the pain was measured using the visual analog scale.

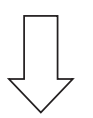

Synovial fluid $(1 \mathrm{ml})$ was drawn from the diseased joint under aseptic precautions.<smiles>C1CC2CC12</smiles>

Level of SOD was estimated using the nitro blue tetrazolium chloride (NBT) reduction method.
Total antioxidant capacity was estimated using the phosphomolybdenum method.
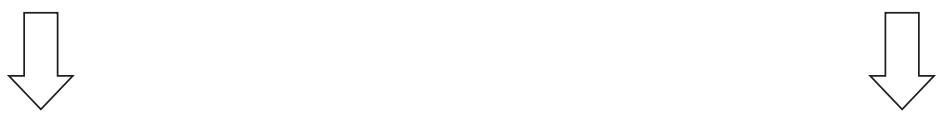

The data obtained was analyzed for statistical significance using spearman's rank correlation method to obtain the correlation coefficient between SOD level and the pain score, and the total antioxidant capacity and the pain score separately.

Results:

Statistics:

Statistical analysis was performed with Statistical Package for Social Science (SPSS) version 11.0 software package. Table 1 represents the data expressed as M ean \pm Standard
Deviation [SD]. The data obtained were analyzed for statistical significance using Spearman's rank correlation method to obtain the correlation coefficient between SOD level and the pain score, and the total antioxidant capacity and the pain score separately.

Table 1 : MEAN \pm SD OF AGE, SOD, TAC AND PAIN SCORE OBTAINED IN THE STUDY GROUP

\begin{tabular}{|l|l|l|l|l|}
\hline VARIABLES $(\mathrm{n}=30)$ & AGE & SUPEROXIDE DISMUTASE LEVEL & TOTALANTIOXIDANT CAPACITY & PAIN SCORE \\
\hline Mean \pm SD & $57.933 \pm 8.485$ & $\mathbf{0 . 0 6 4 7 7} \pm 0.044814$ & $\mathbf{1 2 2 . 5 1 0 0 0} \pm 50.764829$ & $6.70 \pm 1.317$ \\
\hline
\end{tabular}

Table 2 : Correlation of SOD and TAC levels in synovial fluid with pain score using spearman's rank coefficient method

\begin{tabular}{|l|l|l|}
\hline VARIABLE & Spearman's Rho (p) & $\mathbf{p}$-value \\
\hline SUPEROXIDE DISMUTASE LEVEL Vs PAIN SCORE & -0.364 & $\varangle 0.05$ \\
\hline TOTAL ANTIOXIDANT CAPACITY Vs PAIN SCORE & -0.185 & $>0.05$ (NS)* \\
\hline NS*: Not significant
\end{tabular}

Table 2 Shows the correlation of SOD levels and TAC levels in synovial fluid with pain in OA of knee using spearman's rank coefficient method. There is a significant correlation between SOD level and pain score indicated by $\rho=0.364(p \varangle 0.05)$. Also, there is little correlation between TAClevel and pain score, which is not significant, indicated by $\rho=0.185(p>0.05)$. 


\section{SEX DISTRIBUTION SEEN IN OA OF KNEE}

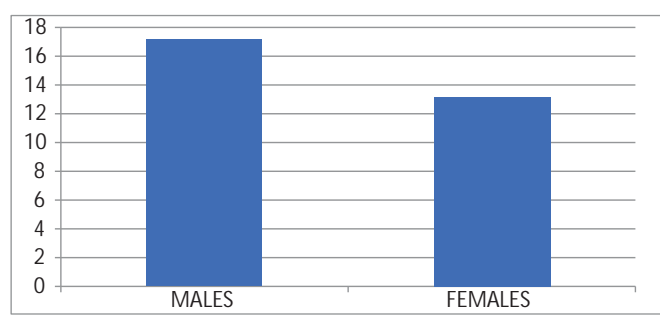

Figure 1 : shows there was male predominance in our study.

\section{AGE DISTRIBUTION SEEN IN OA OF KNEE}

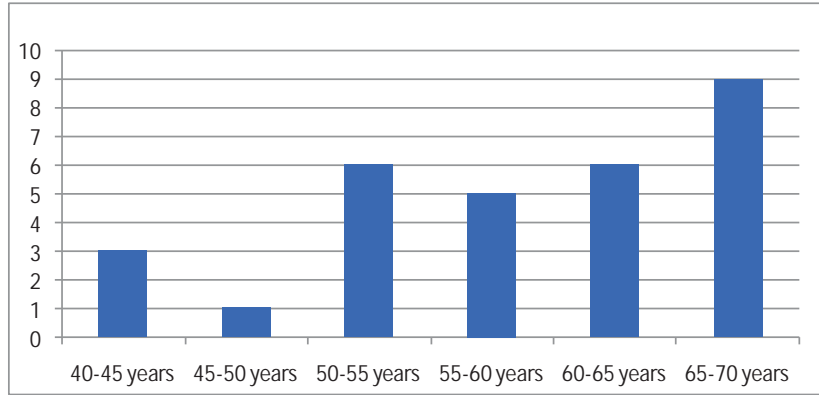

Figure 2 : shows the presence of a direct relationship of $O A$ with increasing age. $M$ ajority of the $O A$ patients included in the study are above 50 years of age. The mean value for the age distribution is $57.933 \pm 8.485$.

SOD LEVELS IN OA OF KNEE (U/ mg protein) EXPRESSED GRAPHICALY

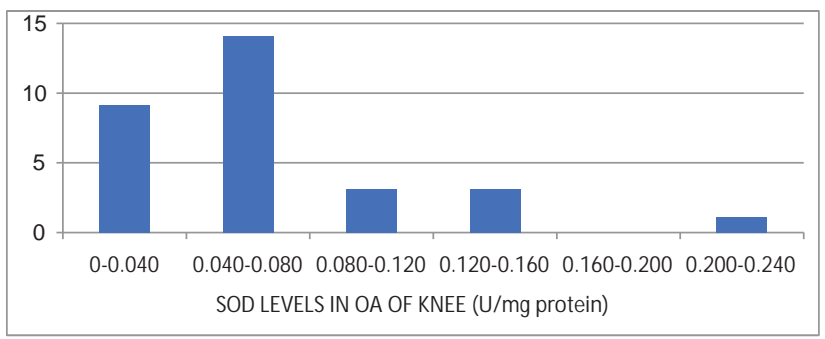

Figure 3: OA patients tend to have a decreased level of SOD in their synovial fluid, due to the increased oxidative stress. This is illustrated in Figure 3, which shows majority of patients having reduced SOD levels with mean SOD value of $0.06477 \pm 0.044814$.

\section{TOTAL ANTIOXIDANT CAPACITY IN OA OF KNEE} $(\mu \mathrm{g} / \mathrm{ml})$ EXPRESSED GRAPHICALY

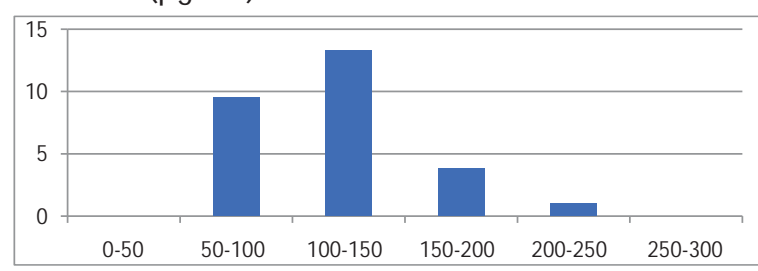

Figure 4 : The normal range of TAC is $167 \pm 22.10$ which is grossly reduced in the samples of synovial fluid collected from the knee of OA patients as per Figure 4. The mean TAC is $122.51000 \pm 50.764829$. This also indicates the increased oxidative stress presents in OA.
PAIN SCORE IN OA OF KNEE

REPRESENTED GRAPHICALLY

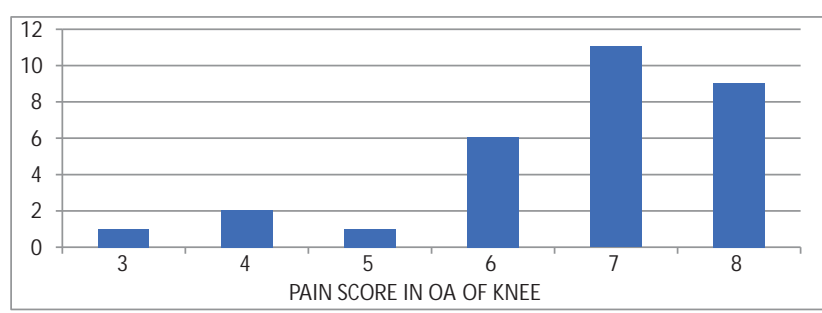

Figure 5 shows an increased intensity of pain in majority of the patients with OA knee. The mean value of pain is $6.70 \pm$ 1.317 suggesting that pain is the most important symptom in $\mathrm{OA}$.

\section{SCATTER DIAGRAM WITH LINE OF REGRESSION: SUPER OXIDE DISM UTASE LEVEL (U/mg protein) V/S PAIN SCORE}

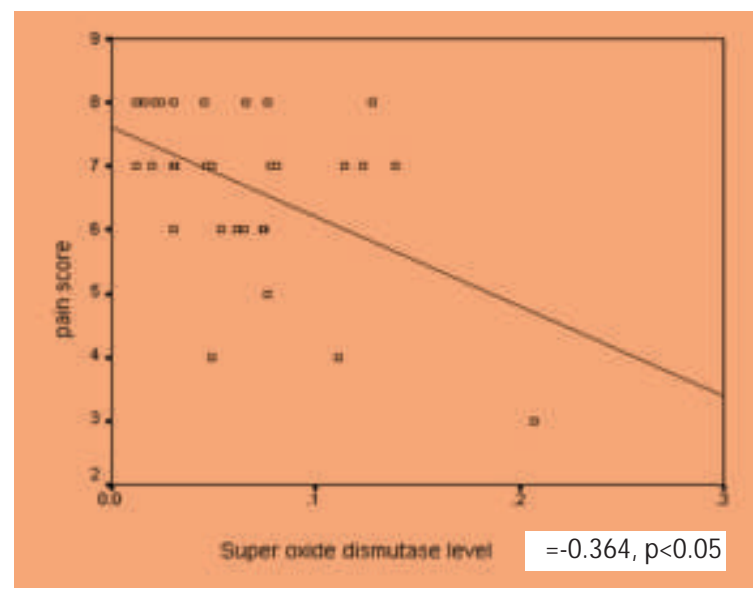

Figure 6 : Scatter diagram of SOD plotted against pain score shows a significant correlation between the two, where $\rho=$ $0.364, p<0.05$.

\section{TOTAL ANTIOXIDANT CAPACITY $(\mu \mathrm{g} / \mathrm{ml})$ V/ S PAIN SCORE}

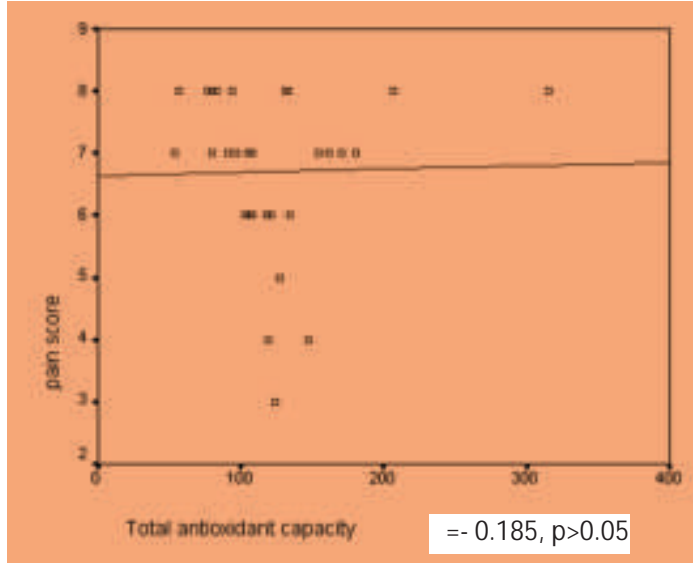

Figure 7: Shows scatter diagram of TAC plotted against pain score which reveals an insignificant relationship between the two with $?=0.185$ and $p>0.05$. 


\section{Discussion :}

The present study examined the correlation between the antioxidant levels in OA knee and the intensity of pain. We demonstrated that both SOD and TAC levels were grossly reduced in majority of the patients. Similar findings were reported in previous studies by Scott JL et al ${ }^{[11]}$ and Regan EA et al ${ }^{[12]}$, where they demonstrated a decreased level of SOD in OA knee. Thus we have established that oxidative stress could be a major etiology of pain in OA. There is excessive production of free radicals caused by the inflammatory mediators like cytokines, proteases and phagocytes. These free radicals heavily tax the antioxidant defense system and cause oxidative stress leads to damage to the cartilage and synovial lining of the joint. When the body is unable to repair the cartilage damage efficiently the joint beginsto degenerate.

The study also verified that $\mathrm{OA}$ is a disease of old age, and is more common in men. With aging, the water content of the cartilage increases, and the protein makeup of cartilage alters. Eventually, cartilage begins to degenerate by flaking or forming tiny crevices. In advanced cases, there is a total loss of cartilage cushion between the bones of the joints. Repetitive use of the worn joints over the years can irritate and inflame the synovium, causing joint pain and swelling. Loss of the cartilage leads to friction between the bone surfaces, leading to pain and limitation of joint mobility. Inflammation of the synovium with increased stress on the subchondral bone stimulates new bone outgrowth (osteophytes) at the margins of the articular surface.

The study illustrated that the pain score on the VAS in majority of patients is high, and thus pain is the most important symptom in $O A$. In the early phase pain is predominantly experienced along with stiffness which increases with strenuous activity.

In our study, we demonstrated the existence of a correlation between the SOD and TAC levels in the synovial fluid and the pain score. The correlation between SOD levels and pain score was significant, while that between TAC levels and pain score was of little significance. Therefore, the present study hypothesized that the pain in
OA patients would be associated with an increased oxidative stress. The association between TAC levels and pain score could not be established possibly because our study group consisted of only $30 \mathrm{OA}$ patients, due to the short duration of the study, which was a major drawback. Another limitation of this study was that no dietary supplements of antioxidants were used to analyze the response following supplementation.

Thus SOD levels in synovial fluid could be a reliable marker to detect early stages of OA. The SOD values can be monitored to attenuate the pain symptoms present in the patients, and thus decrease their disability.

\section{Conclusion:}

The results indicate that there is a positive correlation between the intensity of pain symptoms and SOD levels in the synovial fluid of $O A$ of knee. This significant decrease in the SOD levels in patients with OA is due to the increased free radicals produced by them, which are known mediators of pain. Effective management of SOD levels in the synovial fluid could help in the intervention of pain.

With a thorough study on many other oxidative stress markers and antioxidants among $0 \mathrm{~A}$ patients, the oxidative stress in the synovial fluid can be monitored in the elderly population prone to develop OA.

Also, they can be used as an early marker among the elderly patients and thus by supplementing antioxidants in the diet, future occurrences and complications of OA can be prevented. The study also concludes that further research with the assessment of the effect of antioxidant supplementation in the diet in the regression of pain is required for better understanding of the association between the oxidative stress and pain.

\section{Acknowledgements:}

The authors are grateful to Dr. Sucheta Kumari, Professor in Department of Biochemistry, K.S. Hegde Medical Academy, and the Department of Orthopaedics for providing the necessary facility and full co-operation to carry out the work. We are also grateful to ICMR, for funding the research. 


\section{References:}

1. Lawrence $\mathrm{RC}^{1}$, Helmick CG, Arnett FC, Deyo RA, Felson DT, Giannini EH, Heyse SP, Hirsch R, Hochberg M C, Hunder GG, Liang M H, Pillemer SR, Steen VD,Wolfe F.. Estimates of the prevalence of arthritis and selected musculoskeletal disorders in the United States. Arthritis Rheum.1998;41:778-99.

2. Henrotin Y. and B. Kurz. Antioxidant to treat osteoarthritis: dream or reality? Curr Drug Targets, 2007. 8(2): p. 347-57.

3. Fattman CL, Schaefer LM , Oury TD. Extracellular superoxide dismutase in biology and medicine.Free Radic Biol Med. 2003;35:236-56.

4. Steen V. Petersen, Tim D. Oury, Louise Ostergaard, Zuzana Valnickova, Joanna Wegrzyn, Ida B. Thøgersen,Christian Jacobsen, Russell P. Bowler, Cheryl L. Fattman, James D. Crapo and Jan J. Enghild, Extracellular superoxide dismutase (EC-SOD) binds to type I collagen and protects against oxidative fragmentation. J Biol Chem. 2004;279:13705-10.

5. Oury TD, Chang LY, Marklund SL, Day BJ, Crapo JD. Immunocytochemical localization of extracellular superoxide dismutase in human lung. Lab Invest. 1994;70:889-98.

6. Kinnula VL, Crapo JD. Superoxide dismutases in the lung and human lung diseases. Am J Respir Crit Care M ed. 2003;167:1600-19.

7. KJuul, A Tybjaerg-Hansen, SM arklund, NH Heegaard, R Steffensen, $H$ Sillesen, GJensen, BG Nordestgaard Genetically reduced antioxidative protection and increased ischemic heart disease risk: the Copenhagen City Heart Study. Circulation. 2004;109:59-65.

8. Surapaneni KM, Venkataramana G. Status of lipid peroxidation, glutathione, ascorbic acid, vitamin $\mathrm{E}$ and antioxidant enzymes in patients with osteoarthritis. Indian J M ed Sci 2007; 61:9-14.

9. Sutipornpalangkul W, M orales NP, Charoencholvanich K, Harnroongroj T.Lipid peroxidation, glutathione, vitamin $\mathrm{E}$, and antioxidant enzymes in synovial fluid from patients with osteoarthritis. Int J Rheum Dis. 2009 Dec; 12(4):324-8.

10. M cAlindon, T. E., Jacques, P., Zhang, Y., Hannan, M. T., Aliabadi, P., Weissman, B., Rush, D., Levy, D. and Felson, D. T. Do antioxidant micronutrients protect against the development and progression of knee osteoarthritis? Arthritis \& Rheumatism 1996, 39: 648-656.

11. Scott JL, Gabrieliedes C, Davidson RK, Swingler TE, Clark IM, Wallis GA, Boot-Handford RP, Kirkwood TB, Taylor RW, Young DA. (2010). Superoxide dismutase downregulation in osteoarthritis progression and end-stage diseases. Annals of Rheumatic Disease, 69(9), 9.

12. Regan EA, Bowler RP, Crapo JD. Joint fluid antioxidants are decreased in osteoarthritic joints compared to joints with macroscopically intact cartilage and subacute injury. Osteoarthritis Cartilage. 2008;16:515-521.

13. Beaurchamp, C. and Fridrovich, I. (1971).Superoxide dismutase improved assay applicable to acrylamide gels. Analytical biochemistry. 44, 276-287.

14. Sun, Y., Oberly, L.W. and Li, y. (1998). A simple method for clinical assay of superoxide dismutase. Clinical chemistry. 34, 497-500.

15. Prieto, Manuel, P. and Miguel, A. (1999). Spectrophotometric quantitation of antioxidant capacity through the formation of phosphomolybdenum complex: specific application to the determination of vitamin E. Analytical biochemistry. 259. 337-341. 